\title{
Design, Synthesis, and Biological Evaluation of 4- aminoquinazoline Appended-Benzofuran Hybrids as Epidermal Growth Factor Receptor Inhibitors ${ }^{\dagger}$
}

\author{
Marole Maria Maluleka * and Malose Jack Mphahlele \\ Department of Chemistry, College of Science, Engineering and Technology, University of South Africa, \\ Private Bag X06, Florida 1710, South Africa \\ * Correspondence: 43677541@mylife.unisa.ac.za \\ + Presented at the 2nd Molecules Medicinal Chemistry Symposium (MMCS): Facing Novel Challenges in \\ Drug Discovery, Barcelona, Spain, 15-17 May 2019.
}

Published: 7 August 2019

Nitrogen-containing heterocycles such as quinazolines and benzofurans have received a great amount of interest in targeted therapies as antitumor drugs [1]. Among quinazoline analogues, 4aminoquinazolines have established themselves as selective and effective inhibitors of the epidermal growth factor receptor tyrosine kinase (EGFR-TK), which results from competitive binding at the ATP site [2,3]. We envisaged that molecular hybridization based on condensing $7-$ amino-2-aryl-5-bromobenzofurans with 5-bromo-4-chloroquinazolines in a single molecular framework may provide a more general method for the synthesis of benzofuran-quinazoline hybrids. Retrosynthetic analysis revealed that oximes derived from the 7-acetyl-substituted 2-aryl5-bromobenzofurans would undergo the Beckmann rearrangement followed by hydrolysis of the acetamide derivatives to afford 7-aminobenzofurans for possible condensation with 4chloroquinazolines.

The prepared benzofuran appended 4-aminoquinazoline hybrids were evaluated for cytotoxicity in vitro against human lung cancer (A549), epithelial colorectal adenocarcinoma (Caco$2)$, and hepatocellular carcinoma (C3A) cell lines. Since these tumor cells have been proven to be highly expressed in the cell line of EGFR, we also evaluated representative compounds from each series for potential to inhibit EGFR-TK phosphorylation complemented with molecular docking (in silico) into the ATP binding site of EGFR.

\section{References}

1. Mphahlele, M.J.; Paumo, H.K.; Choong, Y.S. Synthesis and in vitro cytotoxicity of the 4-(halogenoanilino)6-bromoquinazolines and their 6-(4-fluorophenyl) substituted derivatives as potential inhibitors of epidermal growth factor receptor tyrosine kinase. Pharmaceuticals 2017, 10, 87.

2. Mphahlele, M.J.; Mmonwa, M.M.; Aro, A.; McGaw, L.J.; Choong, Y.S. Synthesis, biological evaluation and molecular docking of novel indole-aminoquinazoline hybrids for anticancer properties. Int. J. Mol. Sci. 2018, 19, 2232.

3. Mphahlele, M.J.; Maluleka, M.M.; Aro, A.; McGaw, L.J;; Choong, Y.S. Benzofuran-appended 4aminoquinazoline hybrids as epidermal growth factor receptor tyrosine kinase inhibitors: synthesis, biological evaluation and molecular docking studies. J. Enzyme Inhib. Med. Chem. 2018, 33, 1516-1528. 